\title{
Prediction-Based Resource Allocation for OFDM in Wireless Channels
}

\author{
Kamau Prince, Brian Krongold and Subhrakanti Dey
}

\begin{abstract}
We extend our previous work on optimal dynamic resource allocation in wireless environments to incorporate prediction of the frequency-selective OFDM channel. We briefly summarize our previous work and its exploitation of convexity for the resource allocation problem in point-to-point digital wireless communication links. We introduce channel prediction to overcome latency associated with symbol recovery, channel estimation, and channel-state feedback, which previously restricted resource allocation algorithms to implementation in slowly-fading channel environments. The resourceallocation framework is augmented with channel prediction functionality, and we demonstrate its use with a channel model exhibiting frequency-selective fading with a limited time autocorrelation. Results are presented, illustrating successful implementation, and we conclude with an outline of the course of future investigation to make channelprediction-based resource allocation a viable technique in practical OFDM systems.
\end{abstract}

\section{INTRODUCTION}

Orthogonal Frequency Division Modulation (OFDM) systems are being rapidly developed into platforms to service the exploding bandwidth demands in emerging wireless communication networks. Contemporary OFDM implementations include the IEEE 802.11 WiFi specification, as well as the digital video and audio broadcasts in Europe and worldwide.

In such systems, the wideband transmission channel is implemented as a collection of (independent) narrowband sub-channels, each of which conveys a portion of the total payload data. These systems typically utilise a fixed resource allocation scheme, in which the transceiver operates using pre-assigned transmission rate/power levels. This helps account for the dynamic behavior of the wireless environment by allocating overhead power levels so that the received signal levels (in the presence of time-varying channel conditions) are sufficient to provide some desired overall quality of service (QoS) level. Although this performs acceptably for broadcast environments, the nature of mobile communications motivates an alternate approach. The energy storage constraints typically imposed by mobile networking terminals, as well as the point-to-point communications scenario typically encountered motivates the development of an optimised resource allocation scheme.

The authors are with the ARC Special Research Centre for UltraBroadband Information Networks, Affiliated Program of National ICT Australia, University of Melbourne, Australia email: $\{\mathrm{k} . \mathrm{prince}$, bsk, s.dey\}@ee.mu.oz.au.

This work was supported by the Australian Research Council.
We consider point-to-point digital OFDM communications in the presence of slowly-developing frequencyselective fading. We assume that the fading process has a non-zero time coherence, and that zero crosscorrelation is observed in observations of the fading process across different subchannels ${ }^{1}$. We illustrate the exploitation of these properties to predictively perform resource allocation in such environments.

Our consideration of predictive resource allocation arises from the rapid channel quality variations associated with the mobile wireless channel, in which the time required for channel estimation are significant when compared with the coherence time of the channel. This results in outdated channel state information for transmission resource allocation. We exploit the timecorrelation of the fading process and apply Kalman filtering [1] to provide a forecast of the fade values based on previous (noisy) observations of the fading process. This work is presented as an extension of previous work on optimal resource allocation in the presence of fast fading and composite fading in a non-predictive scenario.

The next section briefly describes the resource allocation (or loading) framework and highlights key aspects. We characterise the prediction as a filtering operation, and illustrate the resource allocation problem under such conditions, where the channel is not restricted to be statistically stationary. A $p$-vector Gauss-Markov fading channel model is implemented for evaluation of its performance and results are presented. Performance limitations of our framework are presented, along with an assessment of the consequences of operation in hostile environments. We then discuss important future directions of this preliminary work and offer some conclusions.

\section{LOADING FRAMEWORK}

We model an OFDM channel as a collection of $p$ discrete narrowband subchannels, each of which affects the received signal power, as represented by a timevarying fading coefficient drawn from a statistical fading process. The OFDM channel fade process may be represented by the random vector $\mathbf{h} \in \mathbb{R}^{\mathbf{p}}$ assuming values $\mathbf{h}(\mathbf{n})$, and where $h_{i}(n)$ denotes the value of the gain tap experienced on the $i^{t h}$ subchannel during

\footnotetext{
${ }^{1}$ Whereas uncorrelated assumption this is not true in practice, it serves to simplify our initial model in order to treat each subchannel separately. With correlated slow-fading processes, we can only expect to do better at tracking and predicting the fade provided some information on the bandwidth coherence function
} 
transmission of the $n^{\text {th }}$ symbol. It is assumed that the transmission occurs with negligible inter-carrier and inter-symbol interference.

In order to achieve data transmission over such a network with a particular QoS, it is required that the receiver signal-to-noise ratio (SNR) meets or exceeds a certain threshold $\left(\mathrm{SNR}_{\mathrm{TH}}\right)$, which is dependent upon the modulation scheme employed and selected sub-symbol rate. This phenomenon is exploited by the implementation of adaptive modulation in time-varying channels, in which the modulation rate $R_{i}(n)$ and transmit power $P_{i}(n)$ on the $i^{t h}$ subchannel are selected such that the $\mathrm{SNR}_{\mathrm{TH}}$ associated with the desired QoS will be satisfied. The $\left(R_{i}(n), P_{i}(n)\right)$ operating point selected for the $i^{t h}$ subchannel during the $n^{\text {th }}$ interval depends on the value assumed by the subchannel fade $h_{i}(n)$. It is assumed that the rate assigned for transmission on a given subchannel is selected from some closed set (which may or may not be constrained to integer values) and that it is possible to abandon a given subchannel by the assignment of zero bits. For now, we assume that it is possible to select any non-negative transmit power $P_{i}(n)$ with which to transmit a given sub-symbol, subject to some overall maximum power constraint $P_{\mathrm{MAX}}$. Hence,

$0 \leq P_{i}(n) \leq P_{\mathrm{MAX}}, R_{i}(n) \in\left\{0, r_{1}, \cdots, r_{L-1}\right\}, \forall i, n$

The aim of the resource allocation algorithm is to accept each $\mathbf{h}(\mathbf{n})$ and select the rate/power conditions for transmission of the OFDM symbol $\mathbf{x}(\mathbf{n})$ to achieve the desired QoS. We select error probability $\mathcal{P}_{e}$ as the QoS metric for our analysis ${ }^{2}$. For a single subchannel, the resource allocation is trivial and the maximum allowed rate is bound only by the power constraint $P_{i} \leq P_{\mathrm{MAX}}$, $\mathrm{SNR}_{\mathrm{TH}}\left(r_{j}\right)$ thresholds, and $h_{i}(n)$. Optimisation over the $p$ sub-symbols introduces additional complexity, as each $P_{i}$ is now bound by the sum of the other $\left(P_{j \neq i}\right)$ 's such that $P_{\mathrm{MAX}} \geq P_{\mathrm{T}}:=\sum_{i=1}^{p} P_{i}$. The total rate $R_{\mathrm{T}}$ of the OFDM symbol is defined as $R_{\mathrm{T}}:=\sum_{i=1}^{p} R_{i}$.

Two possible (and popular) approaches to loading an OFDM system are considered: Rate Maximisation and Margin Maximisation. Rate Maximisation Allocation (RMA) aims to maximise the symbol rate subject to total symbol power $P_{\mathrm{MAx}}$ and subchannel error probability $\mathcal{P}_{e, t}$ constraints. Hence,

$$
\max R_{\mathrm{T}} \quad \text { s.t. } \quad P_{\mathrm{T}} \leq P_{\mathrm{MAX}} \quad \text { and } \quad \mathcal{P}_{e, i} \leq \mathcal{P}_{e, t} ; \forall i
$$

For Margin Maximisation Allocation (MMA), the performance margin is defined as the excess SNR achieved during transmission of a digital symbol, relative to the minimum required SNR to achieve a particular $\mathcal{P}_{e, t}$. Since margin represents a scaling of the allocated $P_{i}$ 's, the solution to this problem may be found by evaluating the minimum power allocation required to satisfy the rate

\footnotetext{
${ }^{2} \mathcal{P}_{e}$ may be defined in terms of bit error rate (BER) or the symbol error probability (SEP) or an outage probability.
}

$R_{\mathrm{DES}}$ and $\mathcal{P}_{e, t}$ :

$$
\min P_{\mathrm{T}} \quad \text { s.t. } \quad R_{T}=R_{\mathrm{DES}} \quad \text { and } \quad \mathcal{P}_{e, i} \leq \mathcal{P}_{e, t} ; \forall i
$$

and then scaling the power of this solution appropriately until the total power budget is used. Assuming that the information in each subchannel is equally important, equal margin may be allocated across all subchannels by factor $\varphi=P_{\mathrm{MAX}} / P_{T}$.

The following analysis is detailed in [2]- [3]. We consider the MMA problem defined in (3), and assume a convex rate-power relationship as is true in general. We can use Lagrange multipliers and the Kuhn-Tucker conditions to characterise the optimal solution. The constrained optimisation problem can be converted into an unconstrained optimisation problem (4), where $J(\lambda)$ is the Lagrange cost and $\lambda \geq 0$.

$$
\min J(\lambda)=\sum_{i=1}^{N} P_{i}+\lambda\left(R_{\mathrm{DES}}-\sum_{i=1}^{N} R_{i}\right)
$$

For fixed $\lambda, J(\lambda)$ corresponds to the minimum power required to satisfy some $R_{\mathrm{T}}$, and which is achieved with a same-slope solution $\left(\frac{\partial P_{i}}{\partial R_{i}}=\lambda\right)$ for each subchannel [2]. The algorithm's goal is to find a $\lambda^{*}$ which achieves the target $R_{\mathrm{DES}}$.

As the $r_{j}$ 's are drawn from a discrete set, the (digital) solution may be found by relaxing the solution of the continuous problem to find the appropriate $\left(R_{i}, P_{i}\right)$ values. This discrete relaxation does not alter the convexity of the objective function, and the optimisation for this problem follows as above, except that in our consideration the derivatives are replaced by differentials $d_{i}\left(r_{j}\right)$, the $(\Delta$ power $) /(\Delta$ rate $)$ between adjacent operating points $r_{j-1}$ and $r_{j}$. Each segment of the continuousvalued $\lambda$ range is associated with an operating point solution for target rate,

$$
R_{i}=\left\{\begin{array}{cc}
r_{j}, & d_{i}\left(r_{j}\right) \leq \lambda<d_{i}\left(r_{j+1}\right) \\
0, & \lambda<d_{i}\left(r_{1}\right) .
\end{array}\right.
$$

Considering the $\mathrm{SNR}_{\mathrm{TH}}\left(r_{j}\right)$ to fulfil the target $\mathcal{P}_{e}$ for a particular rate $r_{j}$ as defined by,

$$
S N R_{i}=\frac{P_{i}\left|H_{i}\right|^{2}}{2 \sigma_{i}^{2}}=P_{i} \cdot C N R_{i},
$$

it is noted that the $P_{i}$ required to achieve $\operatorname{SNR}_{\mathrm{TH}}\left(r_{j}\right)$ is inversely proportional to the channel-to-noise ratio, $\mathrm{CNR}_{i}$. Provided that the observed values of $\mathrm{CNR}_{i}$ affect signal transmission independently of other properties of the subchannel, the operating points may be defined in terms of SNR/rate tuples. This modifies the criteria for operating point selection (7), where $\beta\left(r_{j}\right):=d_{i}\left(r_{j}\right)$. $C N R_{i}$.

$$
R_{i}=\left\{\begin{array}{cc}
r_{j}, & \frac{\lambda}{\beta\left(r_{j+1}\right)} \leq \frac{1}{C N R_{i}}<\frac{\lambda}{\beta\left(r_{j}\right)} \\
0, & \frac{\lambda}{\beta\left(r_{1}\right)}<\frac{1}{C N R_{i}} .
\end{array}\right.
$$

These boundaries are no longer subchannel dependent, which greatly reduces the computation load of implementation [3]. For a given $\lambda, L-1$ lookup table 
boundaries may be computed, and $\left(\mathrm{CNR}_{i}\right)^{-1}$ is used to find $R_{i}$.

This simplification depends on an underlying scalability of the desired rate/power points across subchannels, in a unit-fading environment (AWGN). This may be formally stated; A loading problem has scalability across subchannels if the QoS can be written as a function of only $\left(R_{i}=r_{j}\right)$ and $\left(\zeta_{i} \cdot P_{i}\right)$ for all $i, j$, where $\zeta_{i}$ is a known parameter which completely characterizes the exact or statistical nature of each subchannel. If the channel conditions are known, then $\zeta_{i}$ is the $C N R_{i}$ as above. In the case of a fading environment, $\zeta_{i}$ may be related to a statistical parameter of the fading process [3]. In the case where the exact channel conditions are replaced by a reliable estimate, then this estimate may be used to determine the $\zeta_{i}$ parameter.

The loading algorithms in [2]- [3] are not only optimal, but are very computationally efficient with run times exhibiting $O(p)$ complexity. A major factor in the complexity reduction is the scalability property, and this is expected to hold in many predictive scenarios/models as well. In addition, our loading approach offer a lowcomplexity ability to track small or moderate channel changes, which is critical for a predictive channel system.

\section{Channel Prediction}

We consider the adaptation of our framework to integrate channel prediction. In the previous sections, we outlined that the solution to the resource allocation problems (2), (3) could be evaluated by comparing the inverse channel to noise ratio (a linear function of the fade intensity observed on the channel and the observed channel noise power) with the scaled rate/power derivative of the objective function (5).

We assume our OFDM channel can be described as the product of some fast-fading process and one which produces slow fading. We assume that the slow fading process is dominant and independent of the fast-fading process, which is assumed to vary so quickly that it is impossible to track or predict for resource allocation purposes. The effects of the fast-fading process can be averaged out to focus solely on the slow-fading process for our loading problem. The dominant (slow) fading process produces observations which exhibit a timeautocorrelation function that does not rapidly fall to zero. This time-correlation may be exploited by the implementation of predictive filtering to provide a reliable estimate of channel conditions during the future transmission instant of a desired symbol. This estimate may then be used to determine resource allocation parameters for the transmission of this future symbol. The appeal of such a predictive resource allocation is that it allows an appreciable time to lapse between the sampling of the channel state (during which time the resource allocation algorithm amy be executed, and the solution passed from receiver to transmitter node) and the realisation of the predicted channel state, which is essential for implementing adaptive modulation in rapidly-varying channel environments. Such a scheme would adaptively allocate transmission resources such that the solution of the allocation problem would be closely matched with the state assumed by the channel at the symbol transmission instant.

Our aim is to provide reliable estimates of the values to taken by the channel fading process $\mathbf{s}$ during the transmission of a given symbol $\mathbf{x}$, based on prior observations. Recent progress has been made in the application of predictive filters to estimate future values of the channel fading process observed on wireless channels [4], [5]. Linear Recursive-Least-Square error (RLS) filtering was utilised in [4], and Kalman filtering was employed in [5]. We implemented Kalman filtering, as this provides better performance than linear filters in the presence of statistically non-stationary environments, and those with significant added noise [6].

In our OFDM channel model, we assume that perfect receiver phase compensation is possible, or that the receiver is able to remove transmission phasedistortion effects. We assume that the wideband channel experiences time-varying frequency-selective fading and that the fading process affecting this channel exhibits correlation between successive symbol intervals. No long-range dependency is assumed for the fading process. This allows the framework to be applicable across a wide range of fading process models. Additionally, no assumptions will be made at this stage regarding the correlation of the fading processes observed on any pair of subchannels.

We characterise the channel as introducing fading such that the exponent of the actual fade coefficient assumes values derived from a Gauss-Markov random process. We can characterise this exponent as $\mathbf{s}(\mathbf{n}) \in \mathbb{R}^{\mathbf{p}}$, where

$$
\mathbf{s}(\mathbf{n})=\mathbf{A s}(\mathbf{n}-\mathbf{1})+\mathbf{B u}(\mathbf{n})
$$

where $\mathbf{A} \in \mathbb{R}^{\mathbf{p} \times \mathbf{p}}$ is the (known) state transition matrix, $\mathbf{B} \in \mathbb{R}^{\mathbf{p} \times \mathbf{p}}$ is the (known) input gain matrix, which modulates the Gaussian excitation process, $\mathbf{u}(\mathbf{n}) \in \mathbb{R}^{\mathbf{p}}$. Without loss of generality, we assume that $\mathbf{u} \backsim \mathcal{N}(\mathbf{0}, \mathbf{Q})$. The time autocorrelation of $\mathrm{s}$ falls rapidly:

$$
\mathcal{R}_{\mathbf{s}(\tau)}:=\mathbb{E}[\mathbf{s}(\mathbf{n}) \mathbf{s}(\mathbf{n}+\tau)]=\mathbf{0}, \quad \forall|\tau| \geq \mathbf{2}
$$

The initial state vector, $\mathbf{s}(-\mathbf{1}) \backsim \mathcal{N}\left(\mu_{\mathbf{s}}, \mathbf{C}_{\mathbf{s}}\right)$ is also assumed independent of all $\mathbf{u}(\mathbf{n})$. Each realisation $(\mathbf{s}(\mathbf{n}))$ is observed in the presence of a zero-mean additive white noise process $(\mathbf{w})$, the observations $\left(\mathbf{h}(\mathbf{n}) \in \mathbb{R}^{\mathbf{p} \times \mathbf{p}}\right)$ thus given by

$$
\mathbf{h}(\mathbf{n})=\mathbf{D}(\mathbf{n}) \mathbf{s}(\mathbf{n})+\varepsilon(\mathbf{n})
$$

where $\mathbf{D}(\mathbf{n}) \in \mathbb{R}^{\mathbf{p} \times \mathbf{p}}$ is the (known) observation matrix, and the perturbation vector $\varepsilon(\mathbf{n}) \backsim \mathcal{N}(\mathbf{0}, \mathbf{C}(\mathbf{n}))$. The Gaussian nature of the perturbation prevents any direct prediction of $\mathbf{h}(\mathbf{n})$ : the expected value of the predicted 
$\mathbf{h}(\mathbf{n})$ will therefore be derived from a prediction of the $\mathrm{s}$ variables.

Following [7], the minimum-mean-square error (MMSE) estimate of $\mathbf{s}(\mathbf{n})$, denoted $\hat{\mathbf{s}}(n)$, based on previous observations of $\mathbf{x}(\cdot)$ is provided by Kalman filtering:

1) Prediction:

$$
\begin{gathered}
\hat{\mathbf{s}}(n \mid n-1)=\mathbf{A} \hat{\mathbf{s}}(\mathbf{n}-\mathbf{1} \mid \mathbf{n}-\mathbf{1}) \\
\hat{\mathbf{h}}(n \mid n-1)=\mathbf{D} \hat{\mathbf{s}}(\mathbf{n} \mid \mathbf{n}-\mathbf{1})
\end{gathered}
$$

2) Miniumum Prediction MSE (MPMSE) Matrix :

$$
\mathbf{M}(\mathbf{n} \mid \mathbf{n}-\mathbf{1})=\mathbf{A M}(\mathbf{n}-\mathbf{i} \mid \mathbf{n}-\mathbf{1}) \mathbf{A}^{\prime}+\mathbf{B Q B}^{\prime}
$$

3) Kalman Gain Matrix:

$$
\begin{aligned}
\mathbf{K}(\mathbf{n})=\mathbf{M}(\mathbf{n} \mid \mathbf{n}-\mathbf{1}) \mathbf{D}^{\prime}(\mathbf{n}) & \\
& {\left[\mathbf{C}(\mathbf{n})+\mathbf{D}(\mathbf{n}) \mathbf{M}(\mathbf{n} \mid \mathbf{n}-\mathbf{1}) \mathbf{D}^{\prime}(\mathbf{n})\right]^{-\mathbf{1}} }
\end{aligned}
$$

4) Correction:

$$
\begin{aligned}
\hat{\mathbf{s}}(n \mid n)= & \hat{\mathbf{s}}(n \mid n-1)+ \\
& \mathbf{K}(\mathbf{n})[\mathbf{x}(\mathbf{n})-\mathbf{D}(\mathbf{n}) \hat{\mathbf{s}}(\mathbf{n} \mid \mathbf{n}-\mathbf{1})]
\end{aligned}
$$

\section{5) MMSE Matrix :}

$$
\mathbf{M}(\mathbf{n} \mid \mathbf{n})=[\mathbf{I}-\mathbf{K}(\mathbf{n}) \mathbf{D}(\mathbf{n})] \mathbf{M}(\mathbf{n} \mid \mathbf{n}-\mathbf{1})
$$

The algorithm was initialised with $\hat{\mathbf{s}}(-1 \mid-1)=\mu_{s}$ and $\mathbf{M}(-\mathbf{1} \mid \mathbf{- 1})=\mathbf{C}_{\mathrm{s}}$. To implement longer-range prediction, (17) gives the MMSE estimate of $\hat{\mathbf{y}}(d+n)$ $d>0$.

$$
\mathbb{E}[\hat{\mathbf{h}}(n+d) \mid \mathbf{x}(\mathbf{0}), \cdots, \mathbf{x}(\mathbf{n})]=\mathbf{D A}^{\mathbf{d}-\mathbf{1}} \hat{\mathbf{s}}(\mathbf{n}+\mathbf{1})
$$

\section{PREdictive LoAding}

We applied the framework developed above to implement prediction in the presence of Gauss-Markov fading (8), (10). From (9),we observed that the coherence time of such a channel limits the filtering to single-step prediction. Our prediction will therefore be refreshed at each symbol interval. Such rapidly-decaying time correlation would not typically be encountered in practise, but serve to establish reasonable worst-case bounds on the operation of predictive allocation schemes. The increased coherence time of more practical channels would enable longer range prediction to be implemented (17), and would reduce the frequency of the channel estimation and prediction operations.

We considered an OFDM system with $p=128$ subchannels. Channel effects varied the receive power by a factor $\mathbf{h}$, as given in (10). We assumed reception in a unit noise power environment, and thus the values $\mathbf{h}(\mathbf{n})$ also correspond to receiver SNR. For stability, $\mathbf{A}=\mathbf{0 . 9 8 I}$, where $\mathbf{I} \in \mathbb{R}^{\mathbf{p} \times \mathbf{p}}$ is the identity matrix. We also set $\mathbf{B}=\mathbf{I}$, and the observer was given access to all state variables $(\mathbf{D}=\mathbf{I})$. We assumed a worst-case (in terms of computation load) uncorrelated channel fading. Following the Log-Normal shadowing model observed in [8], we selected $\mathbf{u}$ to be a zero mean Gaussian random vector with a variance $\mathbf{Q}=\sigma_{\mathbf{u}_{\mathbf{i}}}^{\mathbf{2}} \mathbf{I}$, where $\sigma_{u_{i}}^{2}=11 \mathrm{~dB}$. The perturbation $\varepsilon$ was also assumed to be a zero-mean uncorrelated Gaussian random vector of length $p$, and was assigned a variance of $10^{-3}$. The Kalman prediction algorithm (11)-(16) was initially established with a unit prediction horizon - the algorithm was given access to variables $\mathbf{u}(\tau)$ and $\mathbf{h}(\tau)(0 \leq \tau \leq n)$ to determine $\mathbf{s}(\mathbf{n}+\mathbf{1})$.

We desired to implement the MMA (3) to achieve a target symbol rate of $R_{\mathrm{T}}=384 \mathrm{bits} / \mathrm{symbol}$, which would require an average value $\mathbb{E}\left[R_{i}\right]=3$ bits. Our target error probability was $\mathcal{P}_{e}=1 \%$. A sample of the results (for only a subset of four subchannels) is shown in Fig. 1.
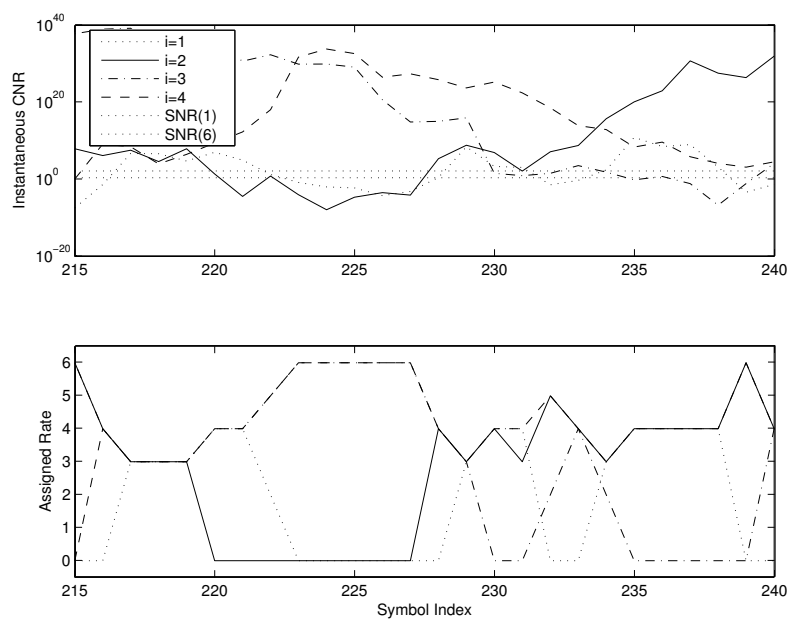

1: Predicted CNR and Resulting Rate Allocation

Fig. 1A (top diagram) shows a sample of the predicted values of the time development of the channel gain conditions observed in four subchannels $(i=1-4)$ (ordinate) plotted against an index of time (abscissa). Fig. 1B (lower diagram) shows the corresponding MMA rate allocation for these subchannels for the same time interval as above. The thresholds indicated by $\operatorname{SNR}(1)$ and $\mathrm{SNR}(6)$ correspond to the $\mathrm{SNR}_{\mathrm{TH}}$ values for $r_{j}=$ 1 and $r_{j}=6$ respectively, at the target $\mathcal{P}_{e}$. Other thresholds were not displayed for the sake of clarity, and lie between these two values. No graphical results were presented for the prediction error process $(\boldsymbol{\Sigma}(\mathbf{n}):=$ $\mathbf{h}(\mathbf{n})-\hat{\mathbf{h}}(\mathbf{n}))$ as its time development resembled a zeromean Gaussian random process with covariance on the order of $10^{-3}$ along the main diagonal, and zero for all off-diagonal elements.

From Fig. 1, we observe the operation of the dynamic MMA scheme, which varied each subsymbol $\left(R_{i}\right)$ to realise the required $R_{\mathrm{T}}$ at the target $\mathcal{P}_{e}$. The subchannel $\left(P_{i}\right)$ 's are scaled to achieve a uniform margin across 
subchannels and hence the values of $P_{i}(n)$ were not presented. It was observed that the changing values observed for $\hat{\mathbf{h}}$ values produced appropriate loading conditions $\left(R_{i}(n), P_{i}(n)\right)$, and that instances of $\hat{h}_{i}(n)<$ SNR(1) resulted in channel abandonment. These and other observations indicate that the MMA is correctly implemented in this environment.

We also noted that the average transmit power required to satisfy similar $\mathcal{P}_{o}$ requirements with fixed resource allocation at an equivalent symbol rate (here, $R_{i}=3 \mathrm{bit}$ ) was much greater the maximum $P_{i}$ values allocated using MMA. These observations indicated correct implementation of predictive resource allocation in OFDM communications over the wireless mobile channel.

\section{ANALYSIS}

\section{A. Prediction Operation}

The Kalman predictor is an implementation of an IIR filter. Steady-state analysis of the predictor operation (following [7]) yields a measure of error performance. From (8), as $n \rightarrow \infty$, the Kalman filter asymptotically becomes an LTI filter provided that $\mathbf{C}(\mathbf{n})=\mathbf{C}$. As $n \rightarrow \infty$ we denote $\mathbf{K}(\infty)$ as the Kalman gain, $\mathbf{M}(\infty)$ as the MMSE, and $\mathbf{M}_{\mathbf{p}}(\infty)$ as the MPMSE (steady-state values). It has been shown that

$$
\begin{aligned}
\mathbf{M}(\infty)=\mathbf{C}\left(\mathbf{A M}(\infty) \mathbf{A}^{\prime}+\mathbf{Q}\right) . \\
\\
\left(\mathbf{A M}(\infty) \mathbf{A}^{\prime}+\mathbf{Q}+\mathbf{C}\right)^{-\mathbf{1}}
\end{aligned}
$$

and that the steady-state transfer function is

$$
\mathcal{H}_{\infty}(z)=\mathbf{K}(\infty)\left(\mathbf{I}-\mathbf{A}(\mathbf{I}-\mathbf{K}(\infty)) \mathbf{z}^{-\mathbf{1}}\right)^{-\mathbf{1}}
$$

The innovation ${ }^{3}$ of the output is therefore,

$$
\begin{aligned}
\tilde{\mathbf{h}} & =\mathbf{h}(\mathbf{n})-\mathbf{I} \hat{\mathbf{s}}(\mathbf{n} \mid \mathbf{n}-\mathbf{1}) \\
& =\mathbf{h}(\mathbf{n})-\mathbf{A} \hat{\mathbf{s}}(\mathbf{n}-\mathbf{1} \mid \mathbf{n}-\mathbf{1}) \mathbf{A}
\end{aligned}
$$

Considering $\hat{\mathbf{s}}(n \mid n)$ (as $n \rightarrow \infty$ ) as the output of a filter with gain $\mathcal{H}_{\infty}(z)$ excited by $\mathbf{x}(\mathbf{n})$, we note that the transfer function relating $\mathbf{h}(\mathbf{n})$ to $\tilde{\mathbf{h}}(n)$ is

$$
\mathcal{H}_{w}(z)=\left(\mathbf{I}-\mathbf{A} \mathbf{z}^{-\mathbf{1}}\right)\left(\mathbf{I}-\mathbf{A}(\mathbf{I}-\mathbf{K}(\infty)) \mathbf{z}^{-\mathbf{1}}\right)^{-\mathbf{1}}
$$

The PSD of $\mathbf{h}(\mathbf{n}), \mathscr{P}_{\mathbf{h h}}(\mathbf{f})$, is given in (22), and the PSD of the prediction filter output ${ }^{4}$ is given by (23).

$$
\begin{gathered}
\mathscr{P}_{h h}(f)=\mathscr{P}_{s s}(f)+\mathbf{C} \\
\mathscr{P}_{\hat{\mathbf{s}} \hat{\mathbf{s}}}(f)=\mathcal{H}_{w}(f) \mathscr{P}_{s s}(f) \mathcal{H}_{w}^{\prime}(f)
\end{gathered}
$$

Denoting $\mathbf{C}_{\tilde{\mathbf{h}}}$ as the covariance of $\tilde{\mathbf{h}}$, the prediction filter error PSD is thus

$$
\mathscr{P}_{\tilde{h} \tilde{h}}(f)=\mathbf{C}_{\tilde{\mathbf{h}}}\left(\mathcal{H}_{\mathbf{w}}(\mathbf{f}) \mathcal{H}_{\mathbf{w}}^{\prime}(\mathbf{f})\right)^{-\mathbf{1}}
$$

As $n \rightarrow \infty$ therefore, the PSD of the filter error becomes flat, and its magnitude is less than the innovation variance [7].

\footnotetext{
${ }^{3}$ INNOVATION is defined as the "new" information in the actual output value; orthogonal to all previous observations of $\mathbf{h}$

${ }^{4}$ where $\mathcal{H}_{w}(f)=\mathcal{H}_{w}(\exp (j 2 \pi f))$.
}

\section{B. Loading Operation}

The reduction of the steady-state PSD allows successful implementation of this method of channel prediction, even in cases where the system parameters are nonstationary (which may be observed when mobile users move through varying environments). For Gaussian inputs, the error of the prediction filter will be a white zeromean Gaussian random vector, having covariance less the covariance of the prediction filter input. It is trivial to show that the predicted value of $\mathbf{x}$ will exceed its actual value approximately half of the time, which will allow the algorithm to allocate resources in a more conservative manner than actual conditions would require, resulting in no QoS violations in the system.

The variance of the predictor error may be minimised when the filter is allowed to sample $\mathbf{h}(\mathbf{n})$ frequently compared to its rate of evolution. This reduces the variance in successive $\mathbf{h}(\mathbf{n})$ values, by reducing the range of possible variation between successive samples, increasing observed correlation. Sampling theory would best guide the selection of an appropriate measurement time horizon for observation of the fading process. Fulfilment of this condition will produce optimal resource allocation.

\section{FUTURE WORK}

This experiment outlined and assessed the performance of a resource optimisation scheme based on single-step prediction of a channel with especially short coherence time. It is straightforward to develop the introductory model introduced in this paper to for longerrange channel prediction, and ultimately for the evaluation of the application of these methods to realistic channel fading data. This will also allow us to accurately determine the actual parameters of the prediction error process $(\boldsymbol{\Sigma})$ and to evaluate the performance of procedures to mitigate error effects on the QoS of the transmission link. Predicting too far ahead will have consequences on performance due to higher prediction error, while a short-range prediction may not still lag the true channel in a practical system with latency. We plan to analyse this tradeoff, and include practical limitations such as a limited amount of noisy subchannel estimates.

We are also investigating the further optimisation of the resource allocation algorithm by the analysis of the time-varying optimal Lagrange parameter $\left(\lambda^{*}(n)\right.$, used to determine the optimal resource allocation scheme for transmission of symbolx $(\mathbf{n})$ ) and the implementation of predictive filtering on this parameter as well. We anticipate that successful implementation of this function will further enhance computational efficiency of the resource-allocation determination.

We are additionally investigating the performance of predictive loading utilising alternate prediction schemes (e.g. Wiener, Linear RLS filtering), in an effort to determine an optimal, or an attractive near-optimal, predictive resource allocation scheme for digital OFDM 
transmission in wireless channels.

\section{CONCLUSION}

We have presented a framework for OFDM resource allocation in a dynamic fading environment with channel prediction. We have demonstrated this model with minimal requirements on the time auto-correlation of the fading process, and no consequential assumptions made on the nature of the cross-correlation of the fading levels observed on individual subchannels.

\section{REFERENCES}

[1] R. E. Kalman, "A new approach to linear filtering and prediction problems," ASME Transactions - Journal of Basic Engineering, vol. 82 , pp. $35-45,1960$.

[2] K. R. B.S Krongold and D. L. Jones, "Computationally efficient optimal power allocation algorithm for multicarrier communication systems," IEEE. Trans. Comun., vol. 48, pp. 23-27, Jan. 2000 .
[3] B. S. Krongold, "New techniques for multicarrier communication systems," Ph.D. dissertation, University of Illinois at UrbanaChampaign, 2001.

[4] S. H. , Alexandra Duel-Hallen and H. Hallen, "Long range prediction of fading signals," IEEE. Signal Processing Magazine, vol. 17 , no. 3, pp. 62-75, May 2000.

[5] M. Sternad and D. Aronsson, "Channel estimation and prediction for adaptive ofdm downlinks," IEEE 58th. Vehicular Technology Conference, vol. 2, pp. 1283-1287, Oct. 2003.

[6] S. Haykin, Adaptive Filter Theory, 4th ed. Upper Saddle River, NJ 07458, USA: Prentice Hall, Inc., 2002.

[7] S. M. Kay, Fundamentals of Statistical Signal Processing: Estimation Theory. Upper Saddle River, NJ 07458, USA: Prentice Hall PTR., 1993.

[8] T. S. Rappaport, Wireless Communications. Upper Saddle River, NJ 07458, USA: Prentice Hall PTR, 2002. 